\title{
TEST IDEALS IN LOCAL RINGS
}

\author{
KAREN E. SMITH
}

\begin{abstract}
It is shown that certain aspects of the theory of tight closure are well behaved under localization. Let $J$ be the parameter test ideal for $R$, a complete local Cohen-Macaulay ring of positive prime characteristic. For any multiplicative system $U \subset R$, it is shown that $J U^{-1} R$ is the parameter test ideal for $U^{-1} R$. This is proved by proving more general localization results for the here-introduced classes of "F-ideals" of $R$ and "F-submodules of the canonical module" of $R$, which are annihilators of $R$ modules with an action of Frobenius. It also follows that the parameter test ideal cannot be contained in any parameter ideal of $R$.
\end{abstract}

Tight closure has produced surprising new results (for instance, that the absolute integral closure of a complete local domain $R$ of prime characteristic is a Cohen-Macaulay algebra for $R$ ) as well as tremendously simple proofs for otherwise difficult theorems (such as the fact that the ring of invariants of a linearly reductive group acting on a regular ring is Cohen-Macaulay). The definition of tight closure is recalled in Section 1, but we refer the reader to the papers of Hochster and Huneke listed in the bibliography for more about its applications. While primarily a prime characteristic notion, tight closure offers insight into arbitrary commutative rings containing $\mathbb{Q}$ by fairly standard "reduction to characteristic $p$ " techniques.

Despite its successes, the tight closure operation, which in its principal setting is a closure operation performed on ideals in a commutative Noetherian ring of prime characteristic, remains poorly understood. For example, tight closure is not known to behave well under localization; given an ideal $I$ of a ring $R$, letting $I^{*}$ denote the tight closure of $I$, it is not known whether or not

$$
I^{*} R_{P}=\left(I R_{P}\right)^{*}
$$

where $R_{P}$ is the localization of $R$ at some prime ideal $P$. Even the simpler question "If all ideals of $R$ are tightly closed, is the same true of $R_{P}$ ?" remains unsolved. Each of these questions has been affirmatively answered in some special cases, but a general proof remains elusive, even when $R$ is a complete local ring. One of the goals of this paper is to show that at least certain significant aspects of the tight closure theory are well behaved with respect to localization.

Received by the editors September 7, 1994.

1991 Mathematics Subject Classification. Primary 13A35; Secondary 13D45, $13 C 13$.

Key words and phrases. Tight closure, test ideals, Frobenius action.

The author is supported by the National Science Foundation. 
Test elements, which are annihilators of tight closure relations (see Definition 4.1), are central to the study of tight closure and its applications. For instance, test elements lie at the heart of C. Huneke's proof of the Uniform Artin-Rees Theorem $[\mathrm{Hu}]$. Test elements and test ideals were introduced by Hochster and Huneke in [HH1], and their further study has been pursued for instance in [HH3] and [HH4]. Ultimately, one would like a theorem which states that if $I$ is the test ideal of $R$, then $I R_{P}$ is the test ideal of the localization $R_{P}$ of $R$. In this case, it would follow that the test ideal defines precisely the (Zariski) closed set of all prime ideals $P$ in Spec $R$ such that $R_{P}$ fails to have all ideals tightly closed.

Restricting attention to the important class of parameter ideals of $R$, one is naturally led to develop a theory of parameter test elements (see Definition 4.3). First introduced in [HH4], parameter test elements were also studied by Velez in his thesis [V], as well as in this author's thesis, where some of the results of this paper first appeared.

This paper presents a theory of parameter test elements by placing the parameter test ideal into the broader context of F-ideals (Definition 3.4). The F-ideals are an interesting class of ideals of a (prime characteristic) ring which by definition are annihilators of submodules of local cohomology modules stable under the action of Frobenius. The parameter test ideal is just one example from the class of F-ideals, a natural class of ideals arising quite independently of specific tight closure questions. The germ of the idea of F-ideals appeared in [S1], where some special cases of the results of the present paper first appeared. This present paper goes further, introducing the related, but somewhat more subtle, idea of an F-submodule of the canonical module for $R$ (see Definition 3.3). Through an understanding of the F-submodules of the canonical module of $R$, we can eventually prove the main results on localization of the parameter test ideal in Section 5.

In Sections 4 and 5, we interpret our results on F-ideals in the specific context of parameter ideals. First in Section 4, we define and develop some basic facts about parameter test ideals, placing them nicely into a framework with the (usual) test ideals. In Section 5, we prove the following localization result.

Theorem 5.2. If $R$ is a complete local Cohen-Macaulay reduced ring with parameter test ideal $J$, the ideal $J U^{-1} R$ will be the parameter test ideal for the ring $U^{-1} R$, where $U$ is any multiplicative system in $R$.

Given the difficulties understanding the behavior of tight closure under localization, Theorem 5.2, which says that parameter test ideals behave well, is an encouraging step. Because the parameter test ideal for a Gorenstein ring turns out to be the same as the test ideal as defined originally by Hochster and Huneke in [HH1], (see the remarks preceding Proposition 4.3), we can deduce some corollaries for test ideals in Gorenstein rings. The result for Gorenstein rings has already been proven in [S1]; the reader is cautioned, however, that in general many results about tight closure that are easy to prove for Gorenstein rings become much harder (and in many cases are still unknown) for an arbitrary Cohen-Macaulay ring.

From Theorem 5.2 we may deduce several corollaries about the local behavior of F-rationality. In particular, it follows that the parameter test ideal defines the locus of non-F-rational points in any complete local ring $R$ (Corollary 5.4), 
so that any element $c \in R$ not in any minimal prime of $R$ has a power which is a parameter test element for $R$ if and only if $R_{c}$ is F-rational (Corollary 5.5). For Gorenstein rings, this says that any any element of $R$ such that $R_{c}$ is F-rational has a power which is a test element for $R$. These corollaries can been proved by different methods (the first results along these lines for Gorenstein rings appear in [HH3], see [V] for more general results). Theorem 5.2 gives insight into why such results should be true.

Section 6 indicates a few more properties of the parameter test ideal (and more generally, any F-ideal) of a Cohen-Macaulay local ring. One notable fact proven here is that the parameter test ideal can never be contained in any ideal generated by a system of parameters. Also, because the actual computation of the test ideal can be difficult, we give a simple example of the computation of a test ideal for a specific ring.

In order to accomplish the goals laid out here concerning the behavior of the F-ideals and F-submodules under localization, we are led to the study of a certain functor "dual to localization" in Section 2 of this paper. This functor is the composition of the functor $\operatorname{Hom}_{R}(-, E(R / m))$ followed by $\operatorname{Hom}_{R}(-, E(R / P))$ where $E(R / P)$ denotes an injective hull of the $R$ module $R / P$ and $P \subset m$ are prime ideals of $R$. This covariant exact functor takes submodules of local cohomology modules with supports in $m$ to submodules of local cohomology modules with supports in $P$. While this form of "colocalization" is interesting in its own right (see, for instance, [Y] or [MS]), its main use for us here is that in prime characteristics, this functor is compatible with the Frobenius map. A study of this compatibility leads to the results on localization of F-ideals, F-submodules, and ultimately, parameter test ideals.

All rings in this paper are commutative, Noetherian rings with unit and are assumed to have prime characteristic $p$.

The author thanks Mel Hochster for many stimulating discussions and Jürgen Herzog whose careful reading and insightful questions exposed mis-statements in an earlier version of parts of this paper.

\section{Preliminaries}

Tight Closure. We first review the definition of tight closure; the reader is referred to [HH1] for more information.

Let $R$ be a commutative Noetherian ring of prime characteristic $p$. Tight closure is a closure operation performed on ideals of $R$, or more generally, on submodules of $R$ modules. For an ideal $I$ of $R$, the tight closure of $I$, denoted $I^{*}$, is the ideal of all elements $z$ for which there exists some $c$ not in any minimal prime of $R$ and some $n \in \mathbb{N}$ such that $c z^{q} \in I^{[q]}$ for all $q=p^{e}$ with $e \geq n$, where $I^{[q]}$ denotes the ideal of $R$ generated by the $q^{\text {th }}$ powers of the elements of $I$.

The definition of the tight closure of a module $N$ in an overmodule $M$, while completely analogous, is somewhat more technical. For any $e \in \mathbb{N}$, we denote by ${ }^{e} R$ the ring $R$ viewed as an $R$ module via the $e^{t h}$ iterate of the Frobenius map $R \longrightarrow{ }^{e} R$ sending $r \longmapsto r^{p^{e}}$. The $e^{t h}$ Peskine-Szpiro functor $F^{e}(*)$ is a covariant functor from the category of $R$ modules to the category of ${ }^{e} R$ modules defined by

$$
F^{e}(M)={ }^{e} R \otimes_{R} M
$$


We have a canonical map $M \longrightarrow F^{e}(M)$ sending $m \longmapsto 1 \otimes m$. For convenience, we denote the image of $m$ under this map by $m^{p^{e}}$ or $m^{q}$, where $q$ is understood to be a power of $p$. When $M=R$, this notation agrees with the natural notation arising from the Frobenius endomorphism. For $R$ modules $N \subset M$, we write $N_{M}^{[q]}$ for the the $R$ submodule of $F^{e}(M)$

$$
\operatorname{Kernel}\left(F^{e}(M) \longrightarrow F^{e}(M / N)\right)=\operatorname{Im}\left(F^{e}(N) \longrightarrow F^{e}(M)\right) \text {. }
$$

Equivalently, $N_{M}^{[q]}$ is the $R$ span in $F^{e}(M)$ of $x^{q}$ where $x \in N$. When $M$ is clear from the context, we often drop the subscript $M$ from the notation $N_{M}^{[q]}$. We now recall the definition of tight closure from [HH1].

1.1. Definition. Let $N \subset M$ be modules over a Noetherian ring $R$ of prime characteristic $p \neq 0$. The element $z \in M$ is in the tight closure of $N$ in $M$, denoted $N_{M}^{*}$, if there exists $c$ not in any minimal prime of $R$ and a nonnegative integer $q_{0}=p^{e_{0}}$ such that

$$
c z^{q} \in N_{M}^{[q]} \text { for all } q=p^{e} \geq q_{0} .
$$

The set of all such $z$ clearly forms a submodule $N_{M}^{*}$ containing $N$. When $M$ is clear from the context, we write simply $N^{*}$ If $N^{*}=N$, we say that $N$ is tightly closed in $\dot{M}$.

1.2. Remark. Virtually all of the theory of tight closure reduces to the case where $R$ is a reduced ring. In particular, if $J$ denotes the nilradical of $R$, then $z \in N_{M}^{*}$ if and only if $\bar{z} \in \bar{N}_{\bar{M}}^{*}$ where $\bar{z}$ is the image of $z$ in $\bar{M}=M / J M$ and $\bar{N}=(N+J M) / J M$. This tight closure is the same whether computed as an $R$ module or as an $R / J$ module.

When $R$ is reduced, we may assume that $q_{0}=1$ (see Proposition 4.1 [HH1] for an easy argument). Of course, if $R$ is a domain, the condition that $c$ not be in any minimal prime of $R$ is simply the condition that $c$ be non-zero.

Some problems arise when we attempt to develop the theory of tight closure of a submodule $N$ in a non-finitely generated module $M$. Some of these problems can be remedied by considering the finitistic tight closure:

$$
N_{M}^{* f g}=\bigcup_{M^{\prime}}\left(N \cap M^{\prime}\right)_{M^{\prime}}^{*}
$$

as $M^{\prime}$ ranges over all finitely generated submodules of $M$. Clearly $N_{M}^{* f g} \subset$ $N_{M}^{*}$, although in general this containment may be strict.

Rings in which all ideals are tightly closed are called weakly F-regular. Because of the unfortunate gap in understanding regarding the closure of this class of rings under localization, we reserve the term $F$-regular for rings in which all ideals are tightly closed and all of whose local rings also have this property. However, many of the interesting applications of the theory of tight closure can be carried over to rings in which only certain ideals are assumed to be tightly closed. In general, the parameter ideals ( $I$ is a parameter ideal if it is generated by height $I$ elements) stand out as a particularly important class of ideals with respect to tight closure. The rings in which all parameter ideals are tightly closed, dubbed the $F$-rational rings in [FW], have distinguished themselves as a particularly interesting class. For instance, they are always normal and Cohen-Macaulay ([HH1]) and even have rational singularities ([S2]). 
Local Cohomology and Matlis Duality. We now recall some standard facts while setting up the notation to be used throughout this paper. A good source for more details on these facts is [BH].

We will be concerned with the "highest" local cohomology of a local ring with support in the maximal ideal, denoted $H_{m}^{d}(R)$, where $d$ is the dimension of $(R, m)$. Local cohomology modules may be described as direct limits of Koszul cohomology. In particular, we recall that for any fixed system of parameters $x_{1}, \ldots, x_{d}$ for the $d$ dimensional local ring $(R, m)$,

$$
H_{m}^{d}(R) \cong \underset{t}{\lim _{t}} \frac{R}{\left(x_{1}^{t}, \ldots, x_{d}^{t}\right)},
$$

where successive maps in this limit system are given by multiplication by $x=$ $\prod_{i=1}^{d} x_{i}$ :

$$
\begin{aligned}
\frac{R}{\left(x_{1}^{t}, \ldots, x_{d}^{t}\right) R} & \longrightarrow \frac{R}{\left(x_{1}^{t+1}, \ldots, x_{d}^{t+1}\right) R}, \\
r+\left(x_{1}^{t}, \ldots, x_{d}^{t}\right) R & \longmapsto x r+\left(x_{1}^{t+1}, \ldots, x_{d}^{t+1}\right) R .
\end{aligned}
$$

It will be convenient to denote an element $\eta \in H_{m}^{d}(R)$ by the equivalence class $\left[z+\left(x_{1}^{t}, \ldots, x_{d}^{t}\right) R\right]$, where $z$ is an element of $R$. Note that $\eta$ is equally well represented by $\left[x z+\left(x_{1}^{t+1}, \ldots, x_{d}^{t+1}\right) R\right]$.

Although the limit system $\lim _{t} \frac{R}{\left(x_{1}^{t}, \ldots, x_{d}^{t}\right)}$ defining $H_{m}^{d}(R)$ is not injective in general, the reader may easily verify that it is injective when $R$ is CohenMacaulay.

Let $P$ be any prime ideal of the Noetherian ring $R$, and $E_{R}\left(\frac{R}{P}\right)$ an injective hull of the $R$ module $R / P$ in the category of $R$ modules. The functor

$$
\operatorname{Hom}_{R}\left(*, E_{R}\left(\frac{R}{P}\right)\right),
$$

denoted also by $*^{\vee} P$, is an exact contravariant functor. For any $R$ module $M$, $M^{\vee} P$ has a unique $R_{P}$ module structure, so that we may view $*^{\vee} P$ as a functor from $R$ modules to $R_{P}$ modules.

When $(R, m)$ is local with maximal ideal $m$, we often consider the case where $P=m$. In this situation, we write $*^{\vee}$ for $*^{\vee} m$ and use $E$ to denote $E_{R}\left(\frac{R}{m}\right)$. Recall that $*^{\vee}$ provides an antiequivalence of the category of Artinian $(R, m)$ modules with the category of Noetherian $\hat{R}$ modules and vice versa. When $M$ is an Artinian $(R, m)$ module, the obvious map $M \rightarrow\left(M^{\vee}\right)^{\vee}$ is an isomorphism; likewise, if $M$ is a Noetherian $R$ module, then the map $M \rightarrow\left(M^{\vee}\right)^{\vee}$ is isomorphic to the map $M \rightarrow \hat{M}$. In particular, $E^{\vee}$ can be identified with $\hat{R}$.

We recall that a canonical module of a local ring $(R, m)$ is defined as any finitely generated $R$ module $\omega_{R}$ whose Matlis dual $\operatorname{Hom}_{R}\left(\omega_{R}, E\right)$ is isomorphic to $H_{m}^{d}(R)$. The canonical module for a local ring, when it exists, is uniquely determined up to (non-unique) isomorphism. When $(R, m)$ is a complete local ring, we may clearly let

$$
\omega_{R}=H_{m}^{d}(R)^{\vee}=\operatorname{Hom}_{R}\left(H_{m}^{d}(R), E\right) .
$$

It is easy to check that if $\omega_{R}$ is a canonical module for $R$, then $\omega_{R} \otimes \hat{R}$ is a canonical module for $\hat{R}$. The reader is warned, however, that there do exist 
rings which do not possess a canonical module. In fact, a Cohen-Macaulay local ring $(R, m)$ possesses a canonical module if and only if $R$ is a homomorphic image of a Gorenstein ring.

The theory of canonical modules is nicest when one restricts attention to the case of a Cohen-Macaulay local ring $(R, m)$. Fix a canonical module $\omega_{R}$ for such $R$. Then $\omega_{R}$ is a Cohen-Macaulay $R$ module and for any prime ideal $P$ of $R$, the $R_{P}$ module $\omega_{R} \otimes_{R} R_{P}$ is a canonical module for $R_{P}$. The map

$$
R \longrightarrow \operatorname{Hom}_{R}\left(\omega_{R}, \omega_{R}\right)
$$

given by multiplication by elements of $R$ is an isomorphism. Moreover, $\omega_{R}$ is isomorphic to a pure height 1 ideal of $R$, which is principal if and only if $R$ is Gorenstein. In particular, for a Gorenstein local ring $R, R$ itself is a canonical module.

Fix a canonical module $\omega$ for the Cohen-Macaulay local ring $(R, m)$ of dimension $d$. Grothendieck's local duality ensures that

$$
H_{m}^{d}\left(\omega_{R}\right) \cong \operatorname{Hom}_{R}\left(\omega_{R}, \omega_{R}\right)^{\vee} \cong R^{\vee} \cong E .
$$

This gives us a specific injective hull of the residue field with which to work; we will henceforth make the identification

$$
\begin{aligned}
& E \cong H_{m}^{d}\left(\omega_{R}\right) \cong H_{m}^{d}(R) \otimes \omega_{R} \\
& \cong \underset{t}{\lim } \frac{R}{\left(x_{1}^{t}, \ldots, x_{d}^{t}\right)} \otimes \omega_{R} \cong \underset{t}{\lim } \frac{\omega_{R}}{\left(x_{1}^{t}, \ldots, x_{d}^{t}\right) \omega_{R}},
\end{aligned}
$$

where $x_{1}, \ldots, x_{d}$ are a fixed system of parameters for $R$ and the maps in the direct limit system are given by multiplication by $x=\prod_{i=1}^{d} x_{i}$. Using the fact that $\omega_{R}$ is Cohen-Macaulay, one may check that the maps in this limit system are all injective. We denote an element $\mu \in E$ by $\left[w+\left(x_{1}^{t}, \ldots, x_{d}^{t}\right) \omega_{R}\right]$, where $w \in \omega_{R}$. Note that under the equivalence relation induced by the direct limit system, $\mu$ may be represented equally well by $\left[x w+\left(x_{1}^{t+1}, \ldots, x_{d}^{t+1}\right) \omega_{R}\right]$.

Actually, the theory of canonical modules can be developed for rings that are not assumed to be Cohen-Macaulay and many of the above statements hold with mild hypothesis [HK]. See also [Ao] or [HH5]. We chose not to pursue this level of generality here.

\section{Some DUAlity Lemmas}

This section contains some duality lemmas that will be crucial to our study of the behavior of F-ideals and F-submodules of the canonical module under localization. We study an exact, covariant functor "dual to localization" which, when restricted to submodules of the highest local cohomology module $H_{m}^{d}(R)$ of $(R, m)$, always produces a submodule of the highest local cohomology module $H_{P R_{P}}^{h t P}\left(R_{P}\right)$ of the localization $R_{P}$. This functor is $(-)^{\stackrel{\vee}{v_{P}}}$, a "double Matlis dual."

We assume throughout that $(R, m)$ is a Cohen-Macaulay local ring possessing fixed a canonical module $\omega$ (e.g. $R$ is complete). We approach the module $H_{m}^{d}(R)$ by considering an action of $\omega_{R}$ on $H_{m}^{d}(R)$ and the annihilator modules that thus arise. 
Fix an injective hull of the residue field of $R$ as described above

$$
E \cong \underset{t}{\longrightarrow} \frac{\omega_{R}}{\left(x_{1}^{t}, \ldots, x_{d}^{t}\right) \omega_{R}}
$$

Using the natural pairing

$$
\omega_{R} \otimes H_{m}^{d}(R) \longrightarrow E=\underset{t}{\lim } \frac{\omega_{R}}{\left(x_{1}^{t}, \ldots, x_{d}^{t}\right) \omega_{R}},
$$

we may think of $\omega_{R}$ as "acting on" $H_{m}^{d}(R)$ : if $w \in \omega_{R}$ and $\eta=[z+$ $\left.\left(x_{1}^{t_{0}}, \ldots, x_{d}^{t_{0}}\right) R\right] \in H_{m}^{d}(R)$, then $w \eta$ will be the image (in $E$ ) of the element $w \otimes \eta$ under this map, namely $\left[z w+\left(x_{1}^{t_{0}}, \ldots, x_{d}^{t_{0}}\right) \omega_{R}\right] \in E$. Note that when $R$ is Gorenstein, so that $\omega_{R}$ may be identified with $R$, this action is the same as the usual $R$ module action of $R$ on $H_{m}^{d}(R)$.

Using this action, we associate to every submodule $N$ of $H_{m}^{d}(R)$ its annihilator submodule $W$ of $\omega_{R}$ :

$$
W=\operatorname{Ann}_{\omega_{R}} N=\left\{w \in \omega_{R} \text { such that } w \eta=0 \text { for all } \eta \in H_{m}^{d}(R)\right\} .
$$

Likewise, given any submodule $W \subset \omega_{R}$, we define an associated annihilator submodule of $H_{m}^{d}(R)$, namely

$$
\operatorname{Ann}_{H_{m}^{d}(R)} W=\left\{\eta=\left[z+\left(x_{1}^{t}, \ldots, x_{d}^{t}\right)\right] \text { such that } w \eta=0 \text { for all } w \in W\right\} \text {. }
$$

We now prove a crucial lemma. Although stated here for Cohen-Macaulay rings, it actually holds for any ring whose completion satisfies Serre's $S_{2}$ condition.

2.1. Lemma. Let $(R, m)$ be a Cohen-Macaulay local ring. Let $N$ be any submodule of $H_{m}^{d}(R)$ and let $W$ be any submodule of $\omega_{\hat{R}}$. The symbol $-_{P}^{V}$ denotes the dual $\operatorname{Hom}_{R}(-, E(R / P))$ where $E(R / P)$ is an injective hull of the $R$ module $R / P$; if the $P$ is omitted, it is assumed to be the maximal ideal $m$.

(i) $N^{\vee} \cong \frac{\omega_{\hat{R}}}{\operatorname{Ann}_{\omega_{\hat{R}}} N}$.

(ii) If $M \subset N$, then $\left(\frac{N}{M}\right)^{\vee} \cong \frac{\operatorname{Ann}_{\omega_{\vec{R}}} M}{\operatorname{Ann}_{\omega_{\vec{R}}} N}$.

(iii) When $R$ is complete, $W=\operatorname{Ann}_{\omega_{R}} N$ if and only if $\operatorname{Ann}_{H_{m}^{d}(R)} W=N$.

(iv) If $R$ is complete and $W=\operatorname{Ann}_{\omega_{R}} N$, then

$$
N^{\vee^{\vee} P}=\operatorname{Ann}_{H_{P R_{P}}^{h p P}\left(R_{P}\right)}\left(W \otimes R_{P}\right) .
$$

In particular, the exact covariant functor $(-)^{\vee^{\vee} P}$ takes submodules of $H_{m}^{d}(R)$ to submodules of $H_{P R_{P}}^{\text {htP }}\left(R_{P}\right)$.

This says that the functor $(*)^{\vee^{\vee} P}$ is an exact covariant functor from submodules of $H_{m}^{d}(R)$ to submodules of $H_{P R_{P}}^{h t P}\left(R_{P}\right)$. We can think of it as "dual to localization" (cf. the remarks following Lemma 2.2).

Proof. (i) Note that $\left(H_{m}^{d}(R)\right)^{\vee}=\omega_{\hat{R}}$. By dualizing the exact sequence

$$
0 \longrightarrow N \longrightarrow H_{m}^{d}(R) \longrightarrow \frac{H_{m}^{d}(R)}{N} \longrightarrow 0,
$$


we see that

$$
N^{\vee} \cong \text { cokernel }\left(\left(\frac{H_{m}^{d}(R)}{N}\right) \stackrel{\vee}{\longrightarrow} \omega_{R} \otimes \hat{R} \cong \omega_{\hat{R}}\right) .
$$

On the other hand, because $\operatorname{Hom}_{R}((*), E)$ is exact,

$$
\left(\frac{H_{m}^{d}(R)}{N}\right) \cong \operatorname{kernel}\left(H_{m}^{d}(R)^{\vee} \longrightarrow N^{\vee}\right),
$$

which consists precisely of those maps in $\operatorname{Hom}_{R}\left(H_{m}^{d}(R), E\right) \cong \omega_{\hat{R}}$ which annihilate $N$. Thus

$$
\left(\frac{H_{m}^{d}(R)}{N}\right)^{\vee} \cong \mathrm{Ann}_{\omega_{R}} N
$$

so that from 2.1.1

$$
N^{\vee} \cong \frac{\omega_{\hat{R}}}{\operatorname{Ann}_{\omega_{\hat{R}}} N}
$$

(ii) Dualizing the exact sequence

$$
0 \longrightarrow M \longrightarrow N \longrightarrow(N / M) \longrightarrow 0 \text {, }
$$

we see that $(N / M)^{\vee}$ is the kernel of either of the isomorphic surjective maps

$$
\left(N^{\vee} \longrightarrow M^{\vee}\right) \cong\left(\frac{\omega_{\hat{R}}}{\mathrm{Ann}_{\omega_{\hat{R}}} N} \longrightarrow \frac{\omega_{\hat{R}}}{\operatorname{Ann}_{\omega_{\hat{R}}} M}\right) .
$$

Thus

$$
\left(\frac{N}{M}\right)^{\vee} \cong \frac{\operatorname{Ann}_{\omega_{R}} M}{\operatorname{Ann}_{\omega_{R}} N}
$$

(iii) Assume that $R$ is complete. In the proof of (i), we showed that

$$
\left(\frac{H_{m}^{d}(R)}{N}\right)^{\vee} \cong \operatorname{Ann}_{\omega_{R}} N
$$

If we denote $\operatorname{Ann}_{\omega_{R}} N$ by $W$, we therefore have an exact sequence

$$
0 \longrightarrow N \longrightarrow H_{m}^{d}(R) \longrightarrow W^{\vee} \longrightarrow 0 \text {. }
$$

Any map $\eta=\left[z+\left(x_{1}, \ldots, x_{d}\right)\right] \in H_{m}^{d}(R)=\operatorname{Hom}_{R}\left(\omega_{R}, E\right)$ gives rise to a map from $W$ to $E$ by restriction:

$$
\begin{gathered}
\eta: \omega_{R} \longrightarrow E \\
w \longmapsto\left[z w+\left(x_{1}, \ldots, x_{d}\right) \omega_{R}\right] .
\end{gathered}
$$

The kernel of this map restricted to $W$ is precisely $\mathrm{Ann}_{H_{m}^{d}(R)} W$, which is isomorphic to $N$ by the exact sequence above.

A similar argument shows the converse.

(iv) We observed in the proof of (i) that we have an exact sequence

$$
0 \longrightarrow N \longrightarrow H_{m}^{d}(R) \longrightarrow W^{\vee} \longrightarrow 0 \text {. }
$$

Thus we have an exact sequence

$$
0 \longleftarrow N^{\vee} \longleftarrow \omega_{R} \longleftarrow W \longleftarrow 0 \text {. }
$$


Applying $\operatorname{Hom}_{R}((*), E(R / P))$ is the same as tensoring with $R_{P}$ and then applying $\operatorname{Hom}_{R_{P}}\left((*), E\left(R_{P} / P R_{P}\right)\right)$, so we have

$$
0 \longrightarrow N^{\vee \vee} \longrightarrow \operatorname{Hom}_{R_{P}}\left(\omega_{R P}, E(R / P)\right) \longrightarrow\left(W \otimes R_{P}\right)^{\vee} P \longrightarrow 0 .
$$

Consequently, $N^{\vee \vee}$ is the kernel of the map

$$
\operatorname{Hom}_{R_{P}}\left(\omega_{R P}, E(R / P)\right) \longrightarrow \operatorname{Hom}_{R_{P}}\left(W \otimes R_{P}, E(R / P)\right)
$$

arising from restriction. As discussed in part (iii), this kernel is

$$
\operatorname{Ann}_{H_{P R_{P}}^{i}\left(R_{P}\right)}\left(W \otimes R_{P}\right) \text {. }
$$

Lemma 2.1 can be interpreted as an analog of the following lemma from [S1]:

2.2. Lemma. Let $(R, m)$ be a local ring and $M$ an arbitrary $R$ module.

(i) If $M$ has $A C C$ or DCC, then $\operatorname{Ann}_{R} M=\operatorname{Ann}_{R}\left(M^{\vee}\right)$.

(ii) $\left(\mathrm{Ann}_{\hat{R}} M\right) \cap R=\mathrm{Ann}_{R} M$ when $M$ is an $\hat{R}$-module.

(iii) When $R$ is complete and $M$ has DCC, $\operatorname{Ann}_{R_{P}}\left(\left(M^{\vee}\right)^{\vee} P\right)=\left(\operatorname{Ann}_{R} M\right) R_{P}$.

(iv) When $M \subset E, M^{\vee} \cong \frac{\hat{R}}{\operatorname{Ann}_{\hat{R}} M}$.

(v) If $N \subset M \subset E$, then $\left(\frac{M}{N}\right)^{\vee} \cong \frac{\operatorname{Ann}_{\hat{R}} N}{\operatorname{Ann}_{\hat{R}} M}$.

For Gorenstein rings, the two lemmas are essentially the same. They differ for an arbitrary (Cohen-Macaulay) ring, however. These lemmas underscore the idea that the functor $\left(*^{\vee}\right)^{\vee} P$ accomplishes for Artinian modules what localization accomplishes for Noetherian modules; we may think of it as "dual to localization". For Artinian $M$, the functor $\left(*^{\vee}\right)^{\vee} P$ vanishes for all nonmaximal $P$ if and only if $M$ has finite length, whereas for Noetherian $M$, the localization functor vanishes for all non-maximal $P$ exactly when $M$ is finite length. For complete $R$, Lemma $2.2(\mathrm{v})$ says that composition functor $\left(*^{\vee}\right)^{\vee} P$ applied to $M \subset E$ amounts to taking the annihilator in an injective hull of the residue field of the local ring $R_{P}$ of the localization (at $P$ ) of the ideal which annihilates $M$. When $M \subset H_{m}^{d}(R)$, the same remark applies, but the annihilator of $M$ is interpreted in $\omega_{R}$.

We will use these observations to compare certain submodules of $H_{m}^{d}(R)$ with submodules of the highest local cohomology modules for local rings of $R$. The point is that the tight closure of the zero module in $H_{m}^{d}(R)$ is a canonically defined submodule of $H_{m}^{d}(R)$ whose annihilator is the parameter test ideal.

The study of functor "dual to localization" is an interesting undertaking in it own right. "Co-localization" has recently been explored for instance in [MS] and $[\mathrm{Y}]$.

\section{The FRobeniUs ACtion on $H_{m}^{d}(R)$ AND DUAL CONCEPTS}

We now wish to develop the theory of F-ideals and F-submodules of the canonical module. We first recall how Frobenius acts on $H_{m}^{d}(R)$. The action of Frobenius on local cohomology was first considered by Peskine and Szpiro, who successfully exploited this action to prove some the "Intersection Conjectures" which had grown out of Serre's work on multiplicities. 
Consider the canonical map

$$
\begin{gathered}
H_{m}^{d}(R) \longrightarrow F^{e}\left(H_{m}^{d}(R)\right)={ }^{e} R \otimes H_{m}^{d}(R) \\
\eta \longmapsto 1 \otimes \eta
\end{gathered}
$$

described in Section 1. Representing $H_{m}^{d}(R)$ as $\underline{\lim }_{t} \frac{R}{\left(x_{1}^{t}, \ldots, x_{d}^{t}\right)}$, we see that

$$
F^{e}\left(H_{m}^{d}(R)\right)=\underset{t}{\lim _{t}}{ }^{e} R \otimes\left(\frac{R}{\left(x_{1}^{t}, \ldots, x_{d}^{t}\right) R}\right) \cong \underset{t}{\lim } \frac{R}{\left(x_{1}^{q t}, \ldots, x_{d}^{q t}\right) R} \cong H_{m}^{d}(R) .
$$

Under the canonical map $H_{m}^{d}(R) \longrightarrow F^{e}\left(H_{m}^{d}(R)\right)$, the element $\eta=[z+$ $\left.\left(x_{1}^{t}, \ldots, x_{d}^{t}\right)\right]$ is sent to

$$
F^{e}(\eta)=\left[z^{q}+\left(x_{1}^{q t}, \ldots, x_{d}^{q t}\right)\right] .
$$

This provides a natural action of Frobenius on $H_{m}^{d}(R)$.

3.1. Definition. A submodule $M$ of $H_{m}^{d}(R)$ is F-stable if $F(M) \subset M$.

We denote by $\langle F(M)\rangle$ the submodule of $H_{m}^{d}(R)$ spanned by the elements of $F(M)$ in $H_{m}^{d}(R)$. Note that when $M$ is F-stable, the fact that $H_{m}^{d}(R)$ has DCC ensures that the descending chain of submodules of $H_{m}^{d}(R)$

$$
M \supset\langle F(M)\rangle \supset\left\langle F^{2}(M)\right\rangle \supset\left\langle F^{3}(M)\right\rangle \supset \ldots
$$

eventually stabilizes. Thus there is some $e$ such that the $R$ submodules of $H_{m}^{d}(R)$ generated by $F^{e^{\prime}}(M)$ and $F^{e}(M)$ are identical for all $e^{\prime} \geq e$.

3.2. Remark. We can interpret the F-stable submodules of $H_{m}^{d}(R)$ in a slightly different way: they are simply the (left) submodules of $H_{m}^{d}(R)$ as a module over the subring $R[F]$ of $\operatorname{End}_{\mathrm{Grp}} R$ generated by $R$ (acting by left multiplication) and by the Frobenius map $F$.

Because F-stable submodules of $H_{m}^{d}(R)$ play an important role in the theory of tight closure (see [S1], [S2]), we are led to consider their duals. Assume throughout that $(R, m)$ is a Cohen-Macaulay local ring of dimension $d$ possessing a canonical module $\omega_{R}$.

By taking annihilators with respect to the pairing $\omega_{R} \times H_{m}^{d}(R) \longrightarrow E$ described in Section 2, we define

3.3. Definition. A module $W \subset \omega_{R}$ is an F-submodule of $\omega_{R}$ if $\operatorname{Ann}_{H_{m}^{d}(R)} W$ is an F-stable submodule of $H_{m}^{d}(R)$.

This can be thought of as an analog of the concept of F-ideals introduced in [S1], which we recall here.

3.4. Definition. An ideal $J$ of $R$ is an F-ideal of $R$ if $\operatorname{Ann}_{H_{m}^{d}(R)} J$ is an F-stable submodule of $H_{m}^{d}(R)$.

Note that F-submodules of $\omega_{R}$ and F-ideals of $R$ can be identified for Gorenstein rings, using the identification of $\omega_{R}$ with $R$. It is this special situation that was exploited in [S1] to prove Theorem 5.2 for Gorenstein rings. However, in order to prove the localization theorem of Section 5, it is the F-submodules of $\omega_{R}$ that are the "right" gadgets to look at. Their study is somewhat more subtle than the study of F-ideals begun in [S1], although many of the ideas are similar. 
3.5. Lemma. For a submodule $W$ of the canonical module $\omega_{R}$ for a local Cohen-Macaulay ring $(R, m)$, the following are equivalent:

(i) $W$ is an F-submodule.

(ii) For all systems of parameters $x_{1}, \ldots, x_{d}$, if any $z \in R$ has the property that $W z \subset\left(x_{1}, \ldots, x_{d}\right) \omega_{R}$, then $W z^{p} \subset\left(x_{1}^{p}, \ldots, x_{d}^{p}\right) \omega_{R}$.

(iii) For some fixed system of parameters $x_{1}, \ldots, x_{d}$, if there exists $z$ and $t \in \mathbb{N}$ with the property that $W z \subset\left(x_{1}^{t}, \ldots, x_{d}^{t}\right) \omega_{R}$, then

$$
W z^{p} \subset\left(x_{1}^{p t}, \ldots, x_{d}^{p t}\right) \omega_{R} .
$$

Proof. (i) $\Longrightarrow$ (ii): Assume that $W$ is an F-submodule of $\omega_{R}$ and that for some system of parameters $x_{1}, \ldots, x_{d}$ for $R$, we have

$$
W z \subset\left(x_{1}, \ldots, x_{d}\right) \omega_{R} \text {. }
$$

This inclusion forces the element $\eta=\left[z+\left(x_{1}, \ldots, x_{d}\right)\right]$ to be in $\operatorname{Ann}_{H_{m}^{d}(R)} W$.

The fact that $W$ is an F-submodule of $\omega_{R}$ means that $\operatorname{Ann}_{H_{m}^{d}(R)} W$ is an F-stable submodule of $H_{m}^{d}(R)$. Thus $F(\eta)=\left[z^{p}+\left(x_{1}^{p}, \ldots, x_{d}^{p}\right)\right]$ is also in $\operatorname{Ann}_{H_{m}^{d}(R)} W$. Hence we see that $W$ kills $F(\eta)$, which is equivalent to $W z^{p} \subset$ $\left(x_{1}^{p}, \ldots, x_{d}^{p}\right) \omega_{R}$, as the direct limit system for $E=H_{m}^{d}\left(\omega_{R}\right)$ is injective.

(ii) $\Longrightarrow$ (iii): Clear.

(iii) $\Longrightarrow$ (i): Fix a system of parameters $x_{1}, \ldots, x_{d}$ for $R$. Represent $H_{m}^{d}(R)$ as $\lim _{t} \frac{R}{\left(x_{1}^{t}, \ldots, x_{d}^{t}\right)}$. In order to show that $W$ is an F-submodule of $\omega_{R}$, we must show that $\mathrm{Ann}_{H_{m}^{d}(R)} W$ is F-stable. Choose any $\eta=[z+$ $\left.\left(x_{1}^{t}, \ldots, x_{d}^{t}\right)\right] \in \mathrm{Ann}_{H_{m}^{d}(R)} W$. This means that $W z \subset\left(x_{1}^{t}, \ldots, x_{d}^{t}\right) \omega_{R}$, whence our hypothesis implies that $W z^{p} \subset\left(x_{1}^{p t}, \ldots, x_{d}^{p t}\right) \omega_{R}$. But then

$$
F(\eta)=\left[z^{p}+\left(x_{1}^{p t}, \ldots, x_{d}^{p t}\right)\right] \in \mathrm{Ann}_{H_{m}^{d}(R)} W
$$

so that $\mathrm{Ann}_{H_{m}^{d}(R)} W$ is F-stable.

A similar statement holds for F-ideals. Because we will need it later, we record it here although it first appeared in [S1].

3.6. Lemma. For an ideal $J$ in a Cohen-Macaulay local ring $(R, m)$, the following are equivalent:

(i) $J$ is an F-ideal.

(ii) For all systems of parameters $x_{1}, \ldots, x_{d}$, if any $z \in R$ has the property that $J z \subset\left(x_{1}, \ldots, x_{d}\right) R$, then $J z^{p} \subset\left(x_{1}^{p}, \ldots, x_{d}^{p}\right) R$.

(iii) For some fixed system of parameters $x_{1}, \ldots, x_{d}$, if there exists $z$ and $t \in \mathbb{N}$ with the property that $J z \subset\left(x_{1}^{t}, \ldots, x_{d}^{t}\right) R$, then $J z^{p} \subset\left(x_{1}^{p t}, \ldots, x_{d}^{p t}\right) R$.

We now show that the property of being an F-submodule of $\omega_{R}$ is well behaved under localization.

3.7. Proposition. Let $P$ be any prime ideal of a local Cohen-Macaulay ring $R$ possessing a canonical module $\omega_{R}$. If $W$ is an F-submodule of $\omega_{R}$, then $W \otimes R_{P}$ is an $F$-submodule of $\omega_{R} \otimes R_{P}$, the canonical module for $R_{P}$.

Proof. Fix $W \subset \omega_{R}$, an F-submodule of $\omega_{R}$. We know that given any system of parameters $x_{1}, \ldots, x_{d}$ for $R$ and $z \in R$ such that $z W \subset\left(x_{1}, \ldots, x_{d}\right) \omega_{R}$, 
then $z^{p} W \subset\left(x_{1}^{p}, \ldots, x_{d}^{p}\right) \omega_{R}$. We wish to show that the submodule $W \otimes R_{P}$ of the canonical module $\omega_{R} \otimes R_{P}=\omega_{R P}$ has the same property with respect to some system of parameters for $R_{P}$. Choose a system of parameters $x_{1}, \ldots, x_{d}$ for $R$ such that the image of the subsequence $x_{1}, \ldots x_{i}$ in $R_{P}$ is a system of parameters for $R_{P}$.

Now suppose that $\frac{z}{1}\left(W \otimes R_{P}\right) \subset\left(\frac{x_{1}}{1}, \ldots, \frac{x_{i}}{1}\right) \omega_{R P}$. There exists some $u \in$ $R-P$ such that $(u z) W \subset\left(x_{1}, \ldots, x_{i}\right) \omega_{R}$. Thus for all nonnegative integers $H$, we have

$$
(u z) W \subset\left(x_{1}, \ldots, x_{i}, x_{i+1}^{H}, \ldots, x_{d}^{H}\right) \omega_{R}
$$

whence

$$
(u z)^{p} W \subset\left(x_{1}^{p}, \ldots, x_{i}^{p}, x_{i+1}^{p H}, \ldots, x_{d}^{p H}\right) \omega_{R}
$$

so

$$
(u z)^{p} W \subset \bigcap_{H>0}\left(x_{1}^{p}, \ldots, x_{i}^{p}, x_{i+1}^{p H}, \ldots, x_{d}^{p H}\right) \omega_{R}
$$

and thus

$$
(u z)^{p} W \subset\left(x_{1}^{p}, \ldots, x_{i}^{p}\right) \omega_{R} .
$$

Tensoring again with $R_{P}$ we see that

$$
\frac{z^{p}}{1}\left(W \otimes R_{P}\right) \subset\left(\frac{x_{1}^{p}}{1}, \ldots, \frac{x_{i}^{p}}{1}\right) \omega_{R P} .
$$

Therefore, by an application of Lemma 3.5, $W \otimes R_{P}$ is an F-submodule of $\omega_{R} \otimes R_{P}$.

Using Lemma 3.6, one can prove along similar lines that if $J$ is an F-ideal in a Cohen-Macaulay local ring $(R, m)$, then $J R_{P}$ is an F-ideal in the localization $R_{P}$ (cf. Proposition 4.8 of [S1]).

3.8. Remark. For a complete local Cohen-Macaulay ring, Lemma 2.1 can be interpreted as saying that the lattice of F-stable submodules of $H_{m}^{d}(R)$ is antiisomorphic to the lattice of F-submodules of $\omega_{R}$. That is, we can attempt to understand the $R[F]$ module structure of $H_{m}^{d}(R)$ by looking at the the Fsubmodules of $\omega_{R}$. Lemma 3.6 gives us a way to study these F-submodules without referring back to $H_{m}^{d}(R)$.

\section{Test ElEMENTS}

In this section we define parameter test elements and the parameter test ideal, realizing the parameter test ideal as a special kind of F-ideal. We first review the definition of and basic facts about test elements from [HH1] and then develop analogs for parameter test elements.

The element " $c$ " that is used in Definition 1.1 of tight closure to "test" for tight closure is allowed to depend on the ideal $I$ and even on the element $z$ we are "testing" for inclusion in $I^{*}$. It is often useful to know that there exist "test elements" $c$ which will work in any tight closure test for $R$.

We record the precise definition of the test ideal and of a test element as in [HH1].

4.1. Definition. The test ideal of $R$ is the ideal of elements $c$ of $R$ such that equivalently:

(1) $c z^{q} \in I^{[q]}$ for all $q=p^{e}$ whenever $z \in I^{*}$ for any ideal $I \subset R$; 
or

(2) $c z \in I$ whenever $z \in I^{*}$ for any ideal $I \subset R$.

An element $c$ of $R$ is said to be a test element if it is in the test ideal but not in any minimal prime of $R$.

Note that in particular, all ideals of $R$ are tightly closed ( $R$ is weakly Fregular) if and only if the test ideal is the unit ideal. Every excellent (e.g. complete) local reduced ring has a test element (this is one of the main results of [HH3]).

The following proposition gives an interesting description of the test ideal for a local ring.

4.2. Proposition. Let $(R, m)$ be a local ring of char $p \neq 0$. Then the test ideal for $R$ is $\operatorname{Ann}_{R}\left(0_{E}^{* f g}\right)$, where $E=E\left(\frac{R}{m}\right)$ is an injective hull of the residue field of $R$.

Proof. See [HH1, Proposition 8.23].

Because of the key role that parameter ideals play in tight closure theory and its applications, one may be interested in knowing what elements will work as "test elements" for this slightly restricted class of ideals. Recall that a parameter ideal is simply an ideal of height $h$ which can be generated by $h$ elements; for excellent local equidimensional (e.g. complete local Cohen-Macaulay) rings, parameter ideals are simply those generated by part of a system of parameters for $R$. We are led naturally to the following definition.

4.3. Definition. The parameter test ideal of $R$ is the ideal of all elements $c \in R$ such that $c I^{*} \subset I$ for all parameter ideals $I$ of $R$ (equivalently, $c z^{q} \in I^{[q]}$ for all $\left.q=p^{e}, e=0,1,2, \ldots\right)$. We say that $c$ is a parameter test element if $c$ is in the parameter test ideal but not in any minimal prime of $R$.

Note that all parameter ideals of $R$ are tightly closed ( $R$ is F-rational) if and only if the parameter test ideal is the unit ideal. Of course the parameter test ideal always contains the test ideal, although in general this containment may be strict. The parameter test ideal was first defined in [HH4].

The next proposition is analogous to Hochster and Huneke's description of the test ideal (Proposition 4.2), placing the theory of test ideals and of parameter test ideals nicely into a similar context. Note that when $R$ is Gorenstein, the following proposition guarantees that the test ideal and the parameter test ideal are one and the same ideal. This recovers the fact that F-regular and F-rational agree for Gorenstein rings ([HH3]).

4.4. Proposition. Let $(R, m)$ be an excellent local ring of dimension $d>0$ and let $J$ be its parameter test ideal.

(i) $J=\left\{c \in R\right.$ such that $c I^{*} \subset I$ for all I generated by a full system of parameters for $R\}$.

(ii) When $R$ is Cohen-Macaulay, $J=\operatorname{Ann}_{R}\left(0_{H_{m}^{d}(R)}^{* f g}\right)=\operatorname{Ann}_{R}\left(0_{H_{m}^{d}(R)}^{*}\right)$.

(iii) When $R$ is Cohen-Macaulay, and $x_{1}, \ldots, x_{d}$ is any fixed system of parameters for $R, J=\left\{c \in R\right.$ such that $c\left(x_{1}^{t}, \ldots, x_{d}^{t}\right)^{*} \subset\left(x_{1}^{t}, \ldots, x_{d}^{t}\right)$ for all $t \in \mathbb{N}\}$.

Proof.

(i) Denote the ideal $\left\{c \in R\right.$ such that $c I^{*} \subset I$ for all $I$ generated by a 
full system of parameters for $R\}$ by $J^{\prime}$. Clearly $J \subset J^{\prime}$. Consider a parameter ideal generated by parameters $x_{1}, \ldots, x_{i}$. We can extend these to a full system of parameters $x_{1}, \ldots, x_{d}$ for $R$. If $z \in\left(x_{1}, \ldots, x_{i}\right) R^{*}$, then $z \in\left(x_{1}, \ldots, x_{i}, x_{i+1}^{H}, \ldots, x_{d}^{H}\right) R^{*}$ for all nonnegative integers $H$. Therefore, for any $c \in J^{\prime}$ we have $c z \in\left(x_{1}, \ldots, x_{i}, x_{i+1}^{H}, \ldots, x_{d}^{H}\right) R$, whence

$$
c z \in \bigcap_{H>0}\left(x_{1}, \ldots, x_{i}, x_{i+1}^{H}, \ldots, x_{d}^{H}\right) R=\left(x_{1}, \ldots, x_{i}\right) R .
$$

Because this holds for any parameter ideal $\left(x_{1}, \ldots, x_{i}\right) R$ and any

$$
z \in\left(x_{1}, \ldots, x_{i}\right) R^{*},
$$

it follows that $c$ is in the parameter test ideal for $R$.

(ii) The point here is that $\eta=\left[z+\left(x_{1}^{t}, \ldots, x_{d}^{t}\right)\right] \in 0_{H_{m}^{d}(R)}^{*}$ if and only if $z \in\left(x_{1}^{t}, \ldots, x_{d}^{t}\right) R^{*}$, so that $\eta \in 0_{\frac{R}{\left(x_{1}^{t}, \ldots, x_{d}^{t}\right)}}^{*} \subset 0_{H_{m}^{d}(R)}^{* f g}$. This is proven in Proposition 3.3 of [S1]. from this, it follows immediately that $0_{H_{m}^{d}(R)}^{*}=0_{H_{m}^{d}(R)}^{* f g}$.

We first demonstrate that $J \subset \operatorname{Ann}_{R}\left(0_{H_{m}^{d}(R)}^{*}\right)$, which requires only that $R$ be equidimensional.

Given any system of parameters $x_{1}, \ldots, x_{d}$ for $R$, we may represent $H_{m}^{d}(R)$ as

$$
\underset{t}{\lim } \frac{R}{\left(x_{1}^{t}, \ldots, x_{d}^{t}\right)} \text {. }
$$

Now if $c \in J$, we wish to show that for any $\eta=\left[z+\left(x_{1}^{t}, \ldots, x_{d}^{t}\right)\right] \in$ $0_{H_{m}^{d}(R)}^{*}$, we have $c \eta=0$. But by Proposition 3.3 of [S1], we know that $\left[z^{+}+\left(x_{1}^{t}, \ldots, x_{d}^{t}\right)\right] \in 0_{H_{m}^{d}(R)}^{*}$ if and only if $z \in\left(x_{1}^{t}, \ldots, x_{d}^{t}\right) R^{*}$, so since $c z \in\left(x_{1}^{t}, \ldots, x_{d}^{t}\right) R$, we have $c \eta=0$.

Conversely, when $R$ is Cohen-Macaulay, given any system of parameters $x_{1}, \ldots, x_{d}$ and $z \in\left(x_{1}, \ldots, x_{d}\right) R^{*}$, we wish to show that $c z \in\left(x_{1}, \ldots, x_{d}\right) R$ whenever $c \in \operatorname{Ann}_{R}\left(0_{H_{m}^{d}(R)}^{*}\right)$. But if $z \in\left(x_{1}, \ldots, x_{d}\right)^{*}$, then $\left[z+\left(x_{1}, \ldots, x_{d}\right)\right]$ $\in 0_{H_{m}^{d}(R)}^{*}$, whence $c\left[z+\left(x_{1}, \ldots, x_{d}\right)\right]=0$. Because the Cohen-Macaulayness of $R$ implies that the limit system

$$
\underset{t}{\lim _{t}} \frac{R}{\left(x_{1}^{t}, \ldots, x_{d}^{t}\right)}
$$

defining $H_{m}^{d}(R)$ is injective, we have that $c z \in\left(x_{1}, \ldots, x_{d}\right) R$, and hence $c \in J$.

(iii) This is immediate from the proof of (ii).

4.5. Proposition. The parameter test ideal of a Cohen-Macaulay local ring is an F-ideal.

Proof. The parameter test ideal of a $d$-dimensional Cohen-Macaulay ring $(R, m)$ is $\operatorname{Ann}_{R} 0_{H_{m}^{d}(R)}^{*}$, by Proposition 4.4. We need to show $0_{H_{m}^{d}(R)}^{*}$ is Fstable. But if $\eta \in 0_{H_{m}^{d}(R)}^{*}$, then there exists $c \in R$ not in any minimal prime such that $c \eta^{[q]}=0\left(c f\right.$. Definition 1.1). So $c\left(\eta^{p}\right)^{[q]}$, which is $c \eta^{[q p]}$ is zero for all $q$, whence $\eta^{p} \in 0_{H_{m}^{d}(R)}^{*}$. 
One may also take the annihilator of $0_{H_{m}^{d}(R)}^{*}$ in $\omega_{R}$ instead of $R$, and think of this as a "parameter test module" for $R$. A similar argument shows that the "parameter test module" is an F-submodule of $\omega_{R}$. In fact, because one can show that $0_{H_{m}^{d}(R)}^{*}$ is the unique maximal F-stable submodule of $H_{m}^{d}(R)$, it follows from the remarks at the end of Section 3 that this test module is the unique minimal F-submodule of $\omega_{R}$.

\section{PARAMETER TeST IDEALS UNDER LOCALIZATION}

We will soon prove the main localization result (Theorem 5.2), which states that parameter test ideals behave well under localization for complete CohenMacaulay rings. This was proven for Gorenstein rings in [S1], using the machinery of F-ideals. To prove the more general result, we must consider both F-ideals and the related machinery of F-submodules of $\omega_{R}$. We first need to prove that F-stable submodules of $H_{m}^{d}(R)$ are preserved under "colocalization."

5.1. Lemma. Let $(R, m)$ be a complete local Cohen-Macaulay ring. If $N \subset$ $H_{m}^{d}(R)$ is F-stable, then $N^{\vee} m^{\vee} P$ is also F-stable.

In other words, functor $(-)^{\vee} m{ }^{\vee} P$ is an exact covariant functor from left $R[F]$ submodules of $H_{m}^{d}(R)$ to left $R_{P}[F]$ submodules of $H_{P R_{P}}^{\text {ht } P}\left(R_{P}\right)$ (cf. Remark 3.2).

Proof. We use the the facts presented in Lemma 2.1. Let $W=\operatorname{Ann}_{\omega_{R}} N$, so that $\mathrm{Ann}_{H_{m}^{d}(R)} W=N$. We have the exact sequence

$$
0 \longrightarrow N \longrightarrow H_{m}^{d}(R) \longrightarrow W^{\vee} \longrightarrow 0
$$

which, by dualizing into $E$, yields the exact sequence

$$
0 \longleftarrow N^{\vee} \longleftarrow \omega_{R} \longleftarrow W \longleftarrow 0
$$

and thus

$$
0 \longleftarrow N^{\vee} \otimes R_{P} \longleftarrow \omega_{R} \otimes R_{P} \longleftarrow W \otimes R_{P} \longleftarrow 0 .
$$

Dualizing over $R_{P}$ into $E_{R_{P}}\left(\frac{R_{P}}{P R_{P}}\right)$, we get

$$
0 \longrightarrow N^{\vee} m^{\vee} P \longrightarrow H_{P R_{P}}^{h t P}\left(R_{P}\right) \longrightarrow\left(W \otimes R_{P}\right)^{\vee} \longrightarrow 0 .
$$

Therefore, by definition, $\operatorname{Ann}_{H_{P R_{P}}^{h t P}\left(R_{P}\right)}\left(W \otimes R_{P}\right)$ is an F-stable submodule of $H_{P R_{P}}^{h t P}\left(R_{P}\right)$, because $W \otimes R_{P}$ is an F-submodule of $\omega_{R P}$. But $A_{n n_{H_{P}}^{h t P}}\left(W \otimes R_{P}\right)$ $=N^{\vee} m^{\vee} P$ by Lemma 2.1 (iv).

Finally, armed with Lemma 5.2 and the duality lemmas of Section 2, we can prove that parameter test ideals are well behaved under localization.

5.2. Theorem. Let $(R, m)$ be a complete local reduced Cohen-Macaulay ring with parameter test ideal $J$. The ideal $J U^{-1} R$ is then the parameter test ideal for the ring $U^{-1} R$, where $U$ is any multiplicative system in $R$.

Proof. We first assume that the multiplicative system is the complement, in $R$, of some prime ideal $P$. 
We first prove that $J R_{P}$ is contained in the parameter test ideal for $R_{P}$. Say $\frac{c}{1} \in J R_{P}$. Fix any system of parameters $x_{1}, \ldots, x_{d}$ for $R$ such that $\frac{x_{1}}{1}, \ldots, \frac{x_{i}}{1}$ is a system of parameters for $R_{P}$ (here dimension $R_{P}=$ height $P=i$ ). By Proposition 4.4 , to check that $\frac{c}{1}$ is a parameter test element for $R_{P}$, it is enough to check that $\frac{c}{1}\left(\frac{x_{1}^{t}}{1}, \ldots, \frac{x_{i}^{t}}{1}\right)^{*} \subset\left(\frac{x_{1}^{t}}{1}, \ldots, \frac{x_{i}^{t}}{1}\right) R_{P}$ for all $t \in \mathbb{N}$.

Because tight closure is known to commute with localization for elements generated by a regular sequence (Theorem 6.9 of $[\mathrm{AHH}]$ ), we see that if $\frac{z}{1} \in$ $\left(\frac{x_{1}^{t}}{1}, \ldots, \frac{x_{i}^{t}}{1}\right) R_{P}^{*}$, then $u z \in\left(x_{1}^{t}, \ldots, x_{i}^{t}\right) R^{*}$ for some $u \in R-P$. Since $c$ is a test element for $R$, we see that $c u z \in\left(x_{1}^{t}, \ldots, x_{i}^{t}\right) R$. This implies that

$$
\frac{c}{1} \cdot \frac{z}{1} \in\left(\frac{x_{1}^{t}}{1}, \ldots, \frac{x_{i}^{t}}{1}\right) R_{P}
$$

for all $t \in \mathbb{N}$ and hence $\frac{c}{1}$ is in the parameter test ideal for $R_{P}$.

To prove the reverse inclusion, we again use Proposition 4.4, which ensures that the parameter test ideal for $R_{P}$ is $\operatorname{Ann}_{R_{P}}\left(0_{H_{P R_{P}}^{i}\left(R_{P}\right)}^{*}\right)$, where $i=$ height $P$. Thus, we need to show that

$$
\operatorname{Ann}_{R_{P}}\left(0_{H_{P R_{P}}^{i}\left(R_{P}\right)}^{*}\right) \subset J R_{P},
$$

where $J=\operatorname{Ann}_{R}\left(0_{H_{m}^{d}(R)}^{*}\right)$ is the parameter test ideal for $R$. Consider

$$
\left(0_{H_{m}^{d}(R)}^{*}{ }^{\vee}\right)^{\vee}=\operatorname{Hom}_{R_{P}}\left(\operatorname{Hom}_{R}\left(0_{H_{m}^{d}(R)}^{*}, E\right), E(R / P)\right) .
$$

By Proposition 5.1, this module is an F-stable submodule of $H_{P R_{P}}^{i}\left(R_{P}\right)$. By Lemma 2.2 (iii), we know that

$$
\operatorname{Ann}_{R_{P}}\left(0_{H_{m}^{d}(R)}^{*}{ }^{{ }^{\prime}}\right)^{\vee} P=J R_{P},
$$

so it will be enough to show that

$$
\left(0_{H_{m}^{d}(R)}^{*}{ }^{\vee} m\right)^{\vee} \subset 0_{H_{P R_{P}}^{i}\left(R_{P}\right)}^{*} .
$$

Since the (honest) test ideal of $R$ is contained in the parameter test ideal $J$ and since $R$ has a test element (all complete local reduced rings do [HH3]), we conclude that there exists some $c \in R$ not in any minimal prime of $R$ such that $c \in J$. But then $\frac{c}{1} \in J R_{P}$ is not in any minimal prime of $R_{P}$ and kills every element of the F-stable submodule $\left(0_{H_{m}^{d}(R)}^{*}{ }^{\vee}\right)^{\vee}$ of $H_{P R_{P}}^{i}\left(R_{P}\right)$. This means that for any $\eta \in\left(0_{H_{m}^{d}(R)}^{*}{ }^{\vee} m\right)^{\vee} P$, also $\eta^{q} \in\left(0_{H_{m}^{d}(R)}^{*}{ }^{\vee} m\right)^{\vee} P$, and $c \eta^{q}=0$ for all such $\eta$. It follows that every element of $\left(0_{H_{m}^{d}(R)}^{*}{ }^{\vee} m\right)^{\vee} P$ is contained in $0_{H_{P R_{P}}^{i}\left(R_{P}\right)}^{*}$, completing the proof of 5.2.2 and hence 5.2.1. We conclude that $J R_{P}$ is the parameter test ideal for $R_{P}$.

We now complete the proof by showing that the theorem remains true when we localize at an arbitrary multiplicative system $U$. We first show that $J U^{-1} R$ is contained in the parameter test ideal for $U^{-1} R$. Say that $I$ is an arbitrary parameter ideal in $U^{-1} R$, and that $z \in I^{*}$ in $U^{-1} R$. These hypotheses are preserved when we localize at any prime ideal of $U^{-1} R$. If $c \in J U^{-1} R$, then 
after passing to $R_{P}, \frac{c}{1} \in J R_{P}$, and by the above argument is a test element for $R_{P}$. Because $\frac{z}{1} \in\left(I R_{P}\right)^{*}$ for all prime ideals $P$ of $U^{-1} R$, we thus see that $\frac{c}{1} \frac{z}{1} \in I R_{P}$ for all primes $P$ in $U^{-1} R$. But then $c z \in I U^{-1} R$ as well, whence $c$ is a parameter test element for $U^{-1} R$.

Conversely, if $c$ is a parameter test element for $U^{-1} R$, then using the argument presented in the first three paragraphs of this proof, we see that the image of $c$ (denoted $\frac{c}{1}$ ) in $R_{P}$ is a parameter test element in every local ring of $U^{-1} R$. As we have already seen that $J R_{P}$ is the parameter test ideal for $R_{P}$, it follows that $\frac{c}{1} \in J R_{P}$ for all primes $P$ in Spec $R$ disjoint from $U$. But then $c \in J U^{-1} R$ as well.

We next record a few immediate corollaries of Theorem 5.2. The first appeared originally in [S1].

5.3. Corollary. If $R$ is a complete local reduced Gorenstein ring with test ideal $J$, then $J U^{-1} R$ is the test ideal for $U^{-1} R$.

Proof. When $R$ is Gorenstein, the parameter test ideal is the same as the test ideal, as evidenced by the fact that $E(R / m) \cong H_{m}^{d}(R)$, and therefore $\operatorname{Ann}_{R}\left(0_{H_{m}^{d}(R)}^{* f g}\right)=\operatorname{Ann}_{R}\left(0_{E}^{* f g}\right)$. By Proposition 4.4, the former is the parameter test ideal for $R$ and by Proposition 4.2 the latter is the test ideal for $R$.

Corollaries 5.4 and 5.5 below are simple consequences of Theorem 5.2.

5.4. Corollary. The non-F-rational locus of a complete local ring $R$ is a closed set defined by the parameter test ideal of $R$.

Proof. Let $J$ be the parameter test ideal for $R$. Then $R_{P}$ is F-rational if and only if the parameter test ideal for $R_{P}\left(=J R_{P}\right)$ is the unit ideal. So $R_{P}$ is non-F-rational if and only if $P$ contains $J$.

5.5. Corollary. If $c \in R$, a complete reduced local Cohen-Macaulay ring, is such that $R_{c}$ is F-rational, then $c$ has a power which is a parameter test element.

Proof. Let $J$ be the parameter test ideal for $R$. If $R_{c}$ is F-rational, then its parameter test ideal, $J R_{c}$ must be the unit ideal. But then $c^{N} \in J$ for some power $N$.

Corollary 5.5 can be thought of as an analog of Theorem 5.10 of [HH3] which states that any element $c$ (not in any minimal prime) of a reduced ring $R$ (which is assumed to be finite over its subring of $p^{\text {th }}$ powers) such that $R_{c}$ is strongly F-regular (e.g. regular) has a power which is a test element for $R$. Velez [V] develops the concept of "strong F-rationality" and proves that (under weak hypothesis) if $R_{c}$ is strongly F-rational, then $c$ has a power which is a parameter test element for $R$. Thus Corollary 5.5 is also proven in [V] (by different methods) for the more general setting of Cohen-Macaulay excellent rings. Velez uses Hochster and Huneke's "Gamma Construction" [HH3] and Neron desingularization to conclude the excellent case from the complete case. It is possible that the same technique would enable one to prove Theorem 5.2 in greater generality, but we do not pursue that here. Of course, Theorem 5.2 is asserting something much stronger than any of the above corollaries. 


\section{SOME FINAL REMARKS ON TEST IDEALS}

We now point out a few interesting properties of F-ideals (and the parameter test ideal in particular) and compute the test ideal in an example.

6.1. Proposition. Let $(R, m)$ be a Cohen-Macaulay local ring. The parameter test ideal for $R$ is not contained in any ideal generated by any system of parameters for $\boldsymbol{R}$. In fact, the only F-ideal contained in a parameter ideal is the zero ideal.

Proof. It suffices to prove the latter claim. If $J$ is an F-ideal, and $J \subset$ $\left(x_{1}, \ldots, x_{d}\right) R$ where $x_{1}, \ldots, x_{d}$ is a system of parameters for $R$, then

$$
I \subset\left(x_{1}, \ldots, x_{d}\right)
$$

implies (by Lemma 3.6, applied with $z=1$ )

$$
I \subset\left(x_{1}^{p}, x_{2}^{p}, \ldots, x_{d}^{p}\right) R .
$$

Repeating we see that

$$
I \subset\left(x_{1}^{q}, x_{2}^{q}, \ldots, x_{d}^{q}\right) R .
$$

for all $q=p^{e}$. But then

$$
I \subset \bigcap_{q}\left(x_{1}^{q}, x_{2}^{q}, \ldots, x_{d}^{q}\right)=0 .
$$

From this property of the parameter test ideal, we see that regular local rings are always F-rational, since the maximal ideal is generated by a system of parameters. Of course, we already know that that regular rings are in fact (strongly) F-regular, but it is interesting to note that the parameter test ideal is forced to be fairly "large" in general.

A Cohen-Macaulay local ring $(R, m)$ of dimension $d$ is said to be $F$-injective is the action of Frobenius on $H_{m}^{d}(R)$ (as described in Section 3) is injective.

6.2. Proposition. Let $(R, m)$ be an F-injective Cohen-Macaulay local ring. Every F-ideal of $R$ is radical. In particular, the parameter test ideal is radical.

Proof. Assume $J$ is an F-ideal and that $y^{N} \in J$. We might as well assume that $N=q=p^{e}$ for some natural number $e$. By definition, $W=\operatorname{Ann}_{H_{m}^{d}(R)} J$ is Fstable. With notation as in Section 1, suppose that $\eta=\left[z+\left(x_{1}^{t_{0}}, \ldots, x_{d}^{t_{0}}\right) R\right] \in$ $W \subset=H_{m}^{d}(R)=\lim _{t} \frac{R}{\left(x_{1}^{t}, \ldots, x_{d}^{t}\right)}$, where $x_{1}, \ldots, x_{d}$ is a fixed system of parameters for $R$. Then $\eta^{q}=\left[z^{q}+\left(x_{1}^{t_{0} q}, \ldots, x_{d}^{t_{0} q}\right) R\right]$ is also in $W$, hence killed by $J$. Thus $(y \eta)^{q}=y^{q} \eta^{q}=0$ in $H_{m}^{d}(R)$ and since Frobenius acts injectively on $H_{m}^{d}(R)$ it follows that $y \eta=0$. Because this argument applies to any $\eta \in W$, we conclude that $y \in J$.

A similar result is that for F-pure rings, the test ideal is always radical [FW]. The computation of tight closure can be quite difficult, even in very simple rings. Knowing the test ideal is often very useful. In the following example, we prove that the test ideal of a certain ring is the maximal ideal. This means that in order to compute the tight closure of an ideal $I$, one need only check elements which are in the socle of $R / I$. 
6.3. An example. The test ideal of

$$
R=\frac{k[x, y, z]}{\left(x^{3}+y^{3}+z^{3}\right)}
$$

is the maximal ideal in all characteristics $p$ (except possibly $p=2,3$ ).

This is easy when $p=1 \bmod 3$ : one may check that $R$ is F-injective, so the test ideal (which is the parameter test ideal in this case) is radical. On the other hand, since $R$ has an isolated singularity, the test ideal is $m$ primary (cf. Corollary 5.5). This argument will not work when $p=2 \bmod 3$, so we give a characteristic free proof.

Proof. Let $J$ denote the test ideal ideal of $R$. First note

$$
\left(x^{3}, y^{3}\right) \subset J
$$

by Theorem 8.23 of [HH4]. This theorem states that if $R$ is module finite and generically smooth over a regular subring $A$, then any element of $A$ which is in the Jacobian ideal for the extension $A \subset R$ is a test element. Applying this with $A=k[x, y]$, where the Jacobian ideal is generated by $3 z^{2}$, we see that So $-z^{3}=x^{3}+y^{3}$ is in the test ideal. Likewise $x^{3}+z^{3}$ and $y^{3}+z^{3}$ are in the test ideal, so $x^{3}, y^{3}$ are in the test ideal.

From Proposition 4.4, we see that an element $c$ is in $J$ if and only if $c\left(x^{t}, y^{t}\right)^{*} \subset\left(x^{t}, y^{t}\right)$ for all $t>>0$. So say $w \in\left(x^{t}, y^{t}\right)^{*}$, where $t \geq 4$. Then both $x^{3} w$ and $y^{3} w$ are in $\left(x^{t}, y^{t}\right)$, so using the fact that $R$ is CohenMacaulay, we see that

$$
w \in\left(x^{t}, y^{t},(x y)^{t-3}\right) .
$$

Write $w=a x^{t}+b y^{t}+v(x y)^{t-3}$. Since $w \in\left(x^{t}, y^{t}\right)^{*}$, one uses "coloncapturing" ([HH1]) to check that $v \in\left(x^{3}, y^{3}\right)^{*}$. But now $c w \in\left(x^{t}, y^{t}\right)$ if and only if $c v \in\left(x^{3}, y^{3}\right)$. This says that $c$ is a test element if and only if

$$
c\left(x^{3}, y^{3}\right)^{*} \subset\left(x^{3}, y^{3}\right)
$$

So what is $\left(x^{3}, y^{3}\right)^{*}$ ? We know that all 6-forms are in here (for instance, by Proposition 3.1 of [S3]). I claim that that's it: there are no forms of lower degree except for the elements already in $\left(x^{3}, y^{3}\right)$. If there were, there'd be a 5 -form. But the 5 -forms not in $\left(x^{3}, y^{3}\right)$ are spanned over $k$ by $x^{2} y^{2} z, x^{2} y z^{2}, x y^{2} z^{2}$. Say

$$
\lambda_{1} x^{2} y^{2} z+\lambda_{2} x^{2} y z^{2}+\lambda_{3} x y^{2} z^{2} \in\left(x^{3}, y^{3}\right)^{*} \text {. }
$$

Using "colon capturing," we can divide out $x y$ to get

$$
u=\lambda_{1} x y z+\lambda_{2} x z^{2}+\lambda_{3} y z^{2} \in\left(x^{2}, y^{2}\right)^{*} .
$$

We leave it to the reader to check (the methods of [S3] are useful) by brute force that $\left(x^{2}, y^{2}\right)^{*}=\left(x^{2}, y^{2}, x y z^{2}\right)$, whence $\left(x^{3}, y^{3}\right)^{*}=\left(x^{3}, y^{3}\right)+(x, y, z)^{6}$. But $m(x, y, z)^{6} \subset\left(x^{3}, y^{3}\right)$, so $J=m$.

In fact, the maximal ideal in the preceding example is the only F-ideal of $R$. For Gorenstein rings $R$, the test ideal is the (unique) minimal F-ideal of $R$, as indicated in the remarks following Proposition 4.5. 


\section{REFERENCES}

[AHH] I. Aberbach, M. Hochster, and C. Huneke, Localization of tight closure and modules of finite phantom projective dimension, J. Reine Angew. Math. 434 (1993), 67-114.

[Ao] Y. Aoyama, Some basic results on canonical modules, J. Math. Kyoto Univ. 23 (1983), 85-94.

[BH] W. Bruns and J. Herzog, Cohen-Macaulay Rings, Cambridge University Press, Cambridge, 1993.

[FW] R. Fedder and K. Watanabe, A characterization of F-regularity in terms of F-purity, Commutative Algebra, MSRI Publications No. 15, Springer-Verlag, New York, 1989, pp. 227-245.

[GH] A. Grothendieck and R. Hartshorne, Local cohomology, Springer-Verlag, New York, 1967.

[HK] J. Herzog and E. Kunz et al., Der kanonische Modul eines Cohen-Macaulay Ringen, Lecture Notes in Math., vol. 238, Springer-Verlag, Berlin, 1971, pp. 35-44.

[HH1] M. Hochster and C. Huneke, Tight closure, invariant theory and the Briançon-Skoda theorem, J. Amer. Math. Soc. 3 (1990), 31-116.

[HH2] _ Infinite integral extensions and big Cohen-Macaulay algebras, Ann. of Math. 135 (1992).

[HH3] _ F-regularity, test elements, and smooth base change, Trans. Amer. Math. Soc. 346 (1995), 1-62.

[HH4] _ Tight closure of parameter ideals and splitting in module finite extensions, J. Algebraic Geom. 3 (1994), 599-670.

[HH5] _ Indecomposable canonical modules and connectedness, Proc. of Summer Research Conference, Mt. Holyoke, 1994.

[Hu] C. Huneke, Uniform bounds in local rings, Invent. Math. 107 (1992), 203-223.

[MS] L. Melkersson and P. Schenzel, The co-localization of an Artinian module, University of Lund and Lund Institute of Technology Preprint series, Lund, Sweden, 1992, pp. 1-10.

[S1] K. E. Smith, Tight closure of parameter ideals, Invent. Math. 115 (1994), 41-60.

[S2] _ F-rational rings have rational singularities, (preprint).

[S3] _ Tight closure in graded rings, (preprint).

[V] J. Velez, Openness of the F-rational locus, smooth base change and Koh's conjecture, Thesis, Univ. of Michigan, 1993.

[W] L. Williams, Uniform stability of kernels of Koszul cohomology indexed by the Frobenius endomorphism, J. Algebra 172 (1995), 721-743.

[Y] Siamak Yassemi, Co-associated primes, Københavns Universitet Mathematisk Institut preprint series, 12, 1993, pp. 1-30.

Department of Mathematics, Massachusetts Institute of Technology, Cambridge, MASSACHUSETTS, 02139

E-mail address: kesmith@math.mit.edu 